\title{
Diffusion of small molecules into medaka embryos improved by electroporation
}

\author{
Gerlinde Jung ${ }^{1}$, Markus Hug ${ }^{1}$, Christian Halter ${ }^{2}$, Andrea Friesenhengst ${ }^{1}$, Johann Walzer ${ }^{2}$ and Thomas Czerny $^{1 *}$
}

\begin{abstract}
Background: Diffusion of small molecules into fish embryos is essential for many experimental procedures in developmental biology and toxicology. Since we observed a weak uptake of lithium into medaka eggs we started a detailed analysis of its diffusion properties using small fluorescent molecules.

Results: Contrary to our expectations, not the rigid outer chorion but instead membrane systems surrounding the embryo/yolk turned out to be the limiting factor for diffusion into medaka eggs. The consequence is a bi-phasic uptake of small molecules first reaching the pervitelline space with a diffusion half-time in the range of a few minutes. This is followed by a slow second phase (half-time in the range of several hours) during which accumulation in the embryo/yolk takes place. Treatment with detergents improved the uptake, but strongly affected the internal distribution of the molecules. Testing electroporation we could establish conditions to overcome the diffusion barrier. Applying this method to lithium chloride we observed anterior truncations in medaka embryos in agreement with its proposed activation of Wnt signalling.

Conclusions: The diffusion of small molecules into medaka embryos is slow, caused by membrane systems underneath the chorion. These results have important implications for pharmacologic/toxicologic techniques like the fish embryo test, which therefore require extended incubation times in order to reach sufficient concentrations in the embryos.
\end{abstract}

Keywords: Medaka, Small molecules, Diffusion, Toxicology, Electroporation, LiCl

\section{Background}

Japanese medaka (Oryzias latipes) are small egg-laying freshwater fish that are native to brackish waters and rice paddies in South-East Asia. Economic husbandry, high fecundity, and ex utero development make them a popular vertebrate model organism in developmental biology and molecular genetics and their transparent chorion facilitates non-invasive observation. Furthermore, they are very hardy and highly resistant to common fish diseases (reviewed in [1]). These properties make medaka ideal for testing of toxic substances [2-5].

Toxicity tests are conducted to evaluate the adverse effects of chemicals or biological substances on organisms and are mainly performed by animal experiments. Even if testing of cosmetic and personal care products has been reduced over the last years, there remain numerous

\footnotetext{
* Correspondence: thomas.czerny@fh-campuswien.ac.at

${ }^{1}$ Department for Applied Life Sciences, University of Applied Sciences, FH

Campus Wien, Helmut-Qualtinger-Gasse 2, A-1030, Vienna, Austria Full list of author information is available at the end of the article
}

chemicals, such as pharmaceuticals or food additives, where animal tests are necessary. On the other hand, several programs like the OECD HPV (High Production Volume) Program or the European Union REACH (Registration, Evaluation, Authorization and Restriction of Chemicals) initiative were introduced to regulate the chemical industry globally (reviewed in [6]). They require extensive test regimes for most chemicals. Without further alternatives, these new regulations would therefore dramatically increase the number of animal experiments, particularly for mammals and birds.

Historically, fish toxicity testing plays an important role in ecotoxicology and aquatic toxicology. It is however a controversial question, whether adult fish can be a replacement for mammals and birds, because they may experience the same levels of pain and distress. One alternative is the use of embryos $[7,8]$. In the fish embryo toxicity (FET) test, newly fertilized eggs are exposed to a chemical for 48 hours and various lethal and sub-lethal endpoints are recorded as described by Lammer and

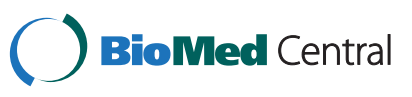


colleagues [9]. Due to their high fecundity and the synchronous extra-uterine development of the transparent embryos, fish are particularly well suited for such a strategy. Most FET tests have been conducted with zebrafish but inter-species comparison with fathead minnow and medaka demonstrated applicability also for other species [10]. Furthermore, fish embryos are suitable for adaption to high throughput protocols [11].

Contrary to zebrafish, medaka embryonic development is relatively long, thus providing extended time for testing. In combination with their hardiness, medaka embryos therefore represent an ideal system for toxicologic/ pharmacologic testing. During FET tests the substances have to diffuse into the developing embryo. Medaka embryos are surrounded by an acellular envelope, the chorion, which protects the developing embryo from environmental influences. Once fertilized, the chorion turns into a rigid structure [12] and might therefore act as an effective barrier that protects the embryo also against chemicals.

Compared to zebrafish, we found a substantially reduced sensitivity for chemicals like lithium chloride for medaka. Closer inspection revealed a limiting diffusion rate of membranes positioned closely to the embryo. Contrary to our expectations, the rigid outer chorion is readily passed by small molecules. In an effort to overcome these problems, we tested the addition of detergents. This effectively improved diffusion, but also affected the internal distribution of the chemicals. Electroporation also enhanced the uptake of fluorescent tracer molecules. When applied to Wnt-signalling, electroporation facilitated the transfer of the GSK-3 inhibitor lithium, thereby inducing deficiencies in medaka anterior-posterior development, similar to those reported for zebrafish [13].

\section{Methods}

\section{Fish stocks and maintenance}

Japanese medaka from the wild-type cab strain were used for this study. Adult fish were maintained at $26^{\circ} \mathrm{C}$ with an artificial 14 hours light and 10 hours dark cycle. Stages were determined according to Iwamatsu [14].

\section{Dechorionation using hatching enzyme}

For dechorionation, the embryos were placed in a small drop of water on a plastic surface. Excess liquid was removed and hatching enzyme was added (10 $\mu$ l hatching enzyme per 15 embryos). The embryos were incubated at $27^{\circ} \mathrm{C}$ until holes appeared in the chorion. The remaining chorion was removed using forceps and the embryos were kept in ERM $(17 \mathrm{mM} \mathrm{NaCl} ; 0.4 \mathrm{mM} \mathrm{KCl}$; $0.27 \mathrm{mM} \mathrm{CaCl}_{2} ; 0.65 \mathrm{mM} \mathrm{MgSO}_{4}$ ) in dishes coated with agarose.

Hatching enzyme was prepared as follows: embryos with visible hatching glands were homogenized in pre- cooled PBS (0.75 $\mu$ l PBS per embryo), incubated over night at $4^{\circ} \mathrm{C}$ and debris was removed by centrifugation (15.000 rpm, $4^{\circ} \mathrm{C}, 10$ minutes). The hatching enzyme solution was stored in aliquots at $-20^{\circ} \mathrm{C}$.

\section{Diffusion experiments}

Sodium fluorescein (Roth; $10 \mathrm{mg} / \mathrm{ml}$ ), rhodamine B (Roth; $10 \mathrm{ng} / \mathrm{ml}$ ) and acridine orange (Roth; $1 \mu \mathrm{g} / \mathrm{ml}$ ) solutions were prepared in 1x Yamamoto's medium (0.128 M NaCl; $0.27 \mathrm{mM} \mathrm{KCl} ; 0.14 \mathrm{mM} \mathrm{CaCl}$;0.24 mM $\mathrm{NaHCO}_{3}$ ). Four embryos were incubated in $50 \mu \mathrm{l}$ staining solution for 40 minutes (if not mentioned otherwise) at $27^{\circ} \mathrm{C}$ protected from light. Standard post incubation washes were performed with $0.5 \mathrm{ml}$ ERM at room temperature on a shaker. The embryos were washed 3 times with fresh ERM in the first seven minutes and subsequently 10, 20, 30 and 60 minutes after the incubation.

For yolk injections, sodium fluorescein $(10 \mathrm{mg} / \mathrm{ml})$ was injected into the yolk using a microinjector (Eppendorf) and borosilicate glass capillaries (Clark Electromedical Instruments).

For methylene blue diffusion, $0.001 \%$ methylene blue in 1x ERM was used. The embryos were incubated for 10 minutes at $27^{\circ} \mathrm{C}$. Standard washing steps were performed as described above.

\section{Quantification of fluorescence intensity}

Embryos were examined in vivo by fluorescence microscopy using a Nikon Eclipse TS100 inverted microscope equipped with an Infinity 2 camera. Pictures with defined exposure times were taken and the fluorescence intensity was quantified from the pictures with the ImageJ software [15]. The quantification of internal concentrations was performed by measuring the fluorescence intensity at different time points of washing and extrapolating back to time zero (start of washing).

As a reference we used empty cellulose sulphate beads with an average diameter of $730 \mu \mathrm{m}$ [16], which were soaked with different concentrations of the fluorescent dyes for several days to reach equilibration. The beads were shortly washed and then transferred into mineral oil, where they were immediately quantified as described above.

\section{Electroporation}

Electroporation of medaka embryos was performed in $1 \times$ Yamamoto's medium. Prior to electroporation the embryos were pre-incubated with the staining solution for 40 minutes in Gene Pulser ${ }^{\bullet}$ disposable Cuvettes with $0.4 \mathrm{~cm}$ gap width (Bio-Rad) at $27^{\circ} \mathrm{C}$ and then electroporated in the same cuvettes. For better comparison, the control experiments were also performed in cuvettes omitting the electroporation pulses. Electroporation was performed with a home-made device 
consisting of a function generator (sine wave with added DC-voltage and variable modulation depth), a pulse generator (generating a defined number of pulses with varying length), a switch (regulated by the pulse generator, controlling the output of the function generator to the amplifier) and an amplifier connected to the cuvette filled with $100 \mu \mathrm{l} 1 \times$ Yamamoto's medium. An oscilloscope was used to display the signals. The conditions for the experiments were: modulation frequency 1 to $10^{5} \mathrm{~Hz}$, modulation depth 1 ; voltage 5 to $50 \mathrm{~V}$; 1 to 3 pulses with intervals of varying length.

For the experiments mimicking chorion diffusion alone, dead embryos were prepared as follows: fifty embryos at stage 17 were electroporated in $400 \mu \mathrm{l} 1 \times$ Yamamoto's medium using 3 pulses of $60 \mathrm{~ms}$ with $200 \mathrm{~ms}$ interval, $60 \mathrm{~V}, 0.5 \mathrm{~A}$ and $35 \mathrm{kHz}$, followed by 30 minutes incubation in $1 \times \mathrm{ERM}$ at $27^{\circ} \mathrm{C}$.

\section{Lithium chloride}

Embryos at stage 14 were incubated in $100 \mu \mathrm{l} 0.4 \mathrm{M}$ lithium chloride (Roth) solution for 10 minutes at $27^{\circ} \mathrm{C}$, followed by electroporation using the following settings: modulation frequency $330 \mathrm{~Hz}$; voltage $15 \mathrm{~V}$; pulse time $100 \mathrm{~ms} ; 1$ pulse. After diffusion and electroporation the embryos were washed as described before. Embryos were examined in vivo by light microscopy using a Zeiss Stemi 2000-C microscope equipped with an AxioCam HRc camera.

\section{Results}

We previously tested the effects of the Wnt signalling pathway on medaka anterior-posterior development by overexpressing wnt1 [17]. Another well-established way to activate this pathway is the application of the GSK-3 inhibitor lithium [18]. However, when we used conditions established for zebrafish embryos [13] no effects could be observed. Except at very high concentrations (above $1 \mathrm{M}$ ) where a low number of embryos showed phenotypes. We therefore reasoned that the diffusion through the chorion into the embryos was not efficient. In order to understand this problem in more detail and to find possible solutions, we started analysing the diffusion properties of the embryo's chorion and membranes. As small and easily quantifiable molecular tracers we selected fluorescing substances.

\section{Diffusion into medaka embryos}

We compared several fluorescing small molecules. Depending on their molecular properties, they were effective at variable concentrations and differed in their distribution within the egg (embryo/yolk). We finally selected fluorescein, which showed enrichment within the embryo (see below) and therefore appeared well suited as a model for small molecules affecting embryonic development.
To quantify the uptake, we compared fluorescence spectroscopic determination of egg extracts and quantification of live embryos under a fluorescence microscope (measurement of pixel intensity of microscopic pictures, for details see Materials and Methods). Although the spectroscopic measurements were more sensitive, we selected the microscopic determination of fluorescence intensity as a standard quantification method. This allowed us to follow the same individuals during different stages of development and thereby to qualify tissue distribution, phenotypic alterations and survival of the embryos.

To determine the optimal conditions, the embryos were incubated in the staining solution at $27^{\circ} \mathrm{C}$ with variable concentrations of fluorescein for different durations. Best quantifiable results were obtained after $40 \mathrm{~min}$ incubation with $10 \mathrm{mg} / \mathrm{ml}$ fluorescein and $30 \mathrm{~min}$ washing time. Even at considerable higher concentrations $(100 \mathrm{mg} / \mathrm{ml})$, fluorescein did not cause any phenotypic alterations in the embryos. These conditions allowed sensitive quantification of the internalized dye over a wide linear range and the settings were therefore applied to all further diffusion experiments. We first analysed the uptake of fluorescein during medaka embryonic development. At the 1-cell stage the internalized fluorescein was localized almost exclusively in the zygote (Figure 1A) and remained in the proliferating cells during early development (Figure 1B). Also later, fluorescence was detected preferentially in the embryo (Figure 1C). For incubation shortly before hatching the signal became enriched in the embryonic liver (Figure 1D). Measurement of the pixel intensity showed a relatively constant fluorescein uptake throughout all developmental stages, except from the 4-cell stage to early morula where the signal slightly increased (Figure 1E).

We also looked at the fate of fluorescein. For this, embryos at the 1-cell stage were treated as described before, but the fluorescence signal was determined after 4 days of incubation in ERM. This extensive washing removed the majority of fluorescein. However, residual fluorescence was detected in the gallbladder and the liver. Even in bright field pictures (Additional file 1) yellow staining of fluorescein can be seen in the gallbladder. Therefore, the fate of fluorescein in older medaka embryos is comparable to that of mammals [19].

\section{Diffusion barriers within the medaka egg}

Overall, the amount of internalized fluorescein was relatively low. After 40 minutes of in-diffusion between 3 and $9 \mu \mathrm{g} / \mathrm{ml}$ (uniform distribution assumed) were detected in the embryo which represents less than $0.1 \%$ of the external concentration $(10 \mathrm{mg} / \mathrm{ml})$. Due to the high concentration in the supernatant the internal fluorescein concentration could not be quantitated during indiffusion. In order to learn more about the kinetics we therefore analysed the out-diffusion. Several fast washing 

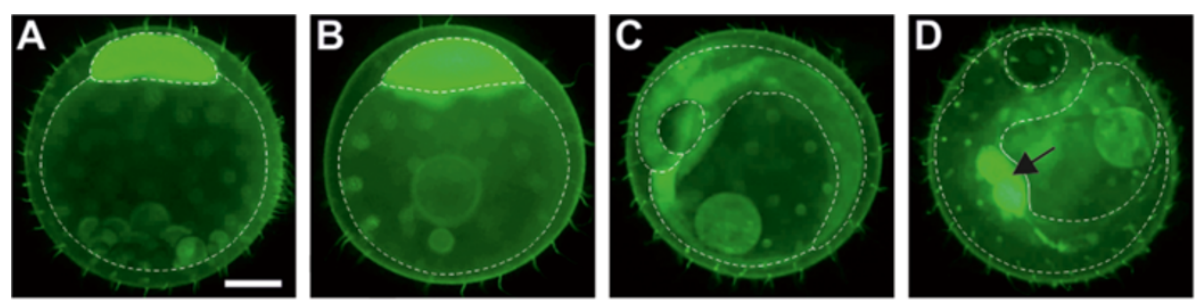

\section{E}

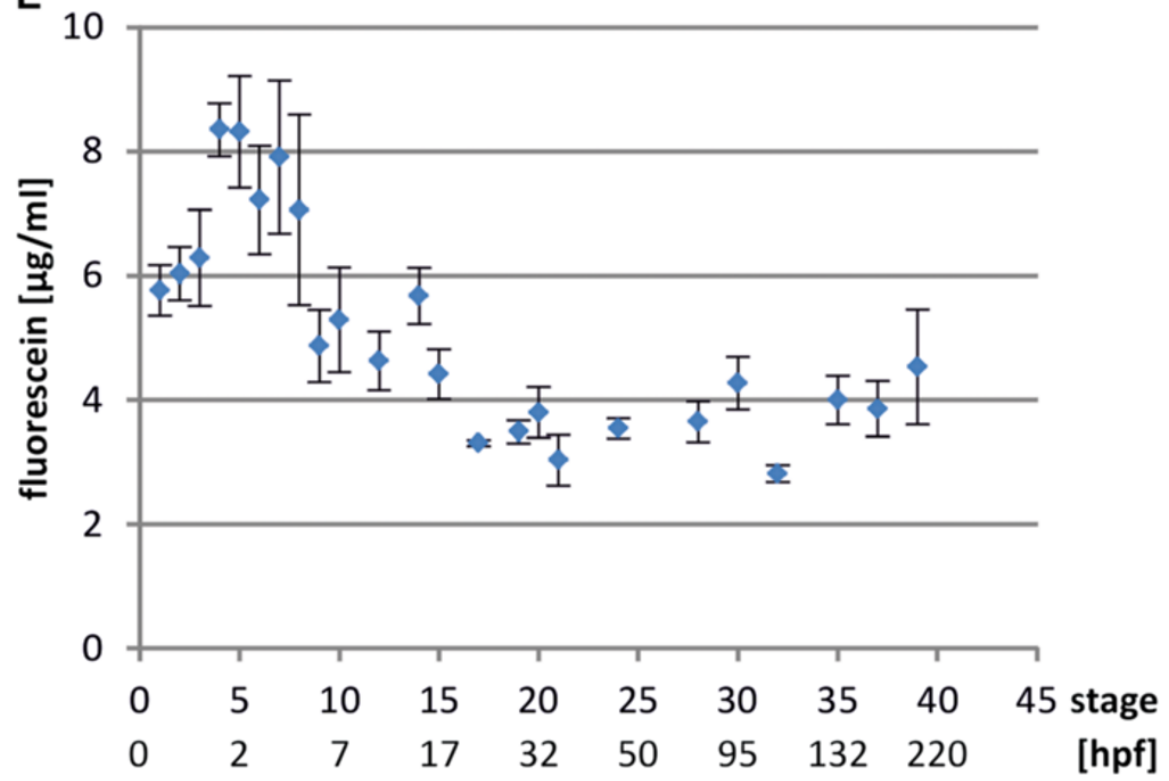

Figure 1 Diffusion of the small molecule fluorescein into the medaka embryo. Embryos at various stages were incubated with $10 \mathrm{mg} / \mathrm{ml}$ fluorescein for 40 minutes at $27^{\circ} \mathrm{C}$. Pictures were taken after 30 minutes of washing and the fluorescence intensity was measured. The dotted lines demarcate the outlines of: $(\mathbf{A}, \mathbf{B})$ yolk and blastoderm, and $(\mathbf{C}, \mathbf{D})$ yolk and embryo. Embryos are shown in lateral view. Black arrow indicates the liver and gall bladder. (A-D) Distribution of fluorescein uptake during four representative embryonic stages: A, stage 2a; B, stage 8; C, stage 26; D, stage 38. The internal concentration of fluorescein (average distribution in embryo/yolk assumed) for different embryonic stages is shown in (E). Scale bar $250 \mu \mathrm{M}$. Abbreviations: hpf, hours post fertilization.

steps directly after the incubation were necessary to remove excess material from the surface of the eggs. First reproducible results were obtained after 7 min of washing (including a 3-fold exchange of the supernatant). Additional measuring points after 20, 30, 60, 90 and 120 minutes revealed two phases for diffusion: a fast loss of signal during the first 30 minutes, followed by a slower second phase (Figure 2A). Calculation of the diffusion half-times for fluorescein resulted in 4 minutes for the first, and 2.4 hours for the second phase.

Based on the bi-phasic out-diffusion observed for fluorescein, we questioned which structures of the medaka egg are responsible for the diffusion kinetics. The egg is shielded by a rigid acellular envelope, the chorion, which represents a prime candidate for a diffusion barrier [3]. However, inside the chorion the embryo and the yolk are covered by additional extra-embryonic membrane systems, which could also affect diffusion. In order to differentiate between these possibilities we manipulated the eggs. In eggs with dead embryos the yolk and membranes shrink to a small ball within the chorion (Figure 2B,C). The quantified fluorescence signal in the egg therefore mainly depends on the diffusion through the chorion. In order to qualify the diffusion properties of the membrane systems covering embryo and yolk, we used hatching enzyme to dechorionate the eggs (Figure 2B,D; for experimental details see Methods).

Quite unexpectedly, diffusion of fluorescein into dead embryos resulted in strong fluorescence (Figure 2C'), indicating that the chorion is readily passed by small molecules. Quantification of the signals (Figure 2E, for details see Methods) showed that the interior concentration almost reached saturation after 40 minutes (50\% after 20 minutes). On the other hand, diffusion into dechorionated embryos was extremely slow, resulting in an average concentration similar to that for intact eggs $(<0.1 \%)$ after 40 minutes (some of the dechorionated embryos showed high signals, which was however due to 

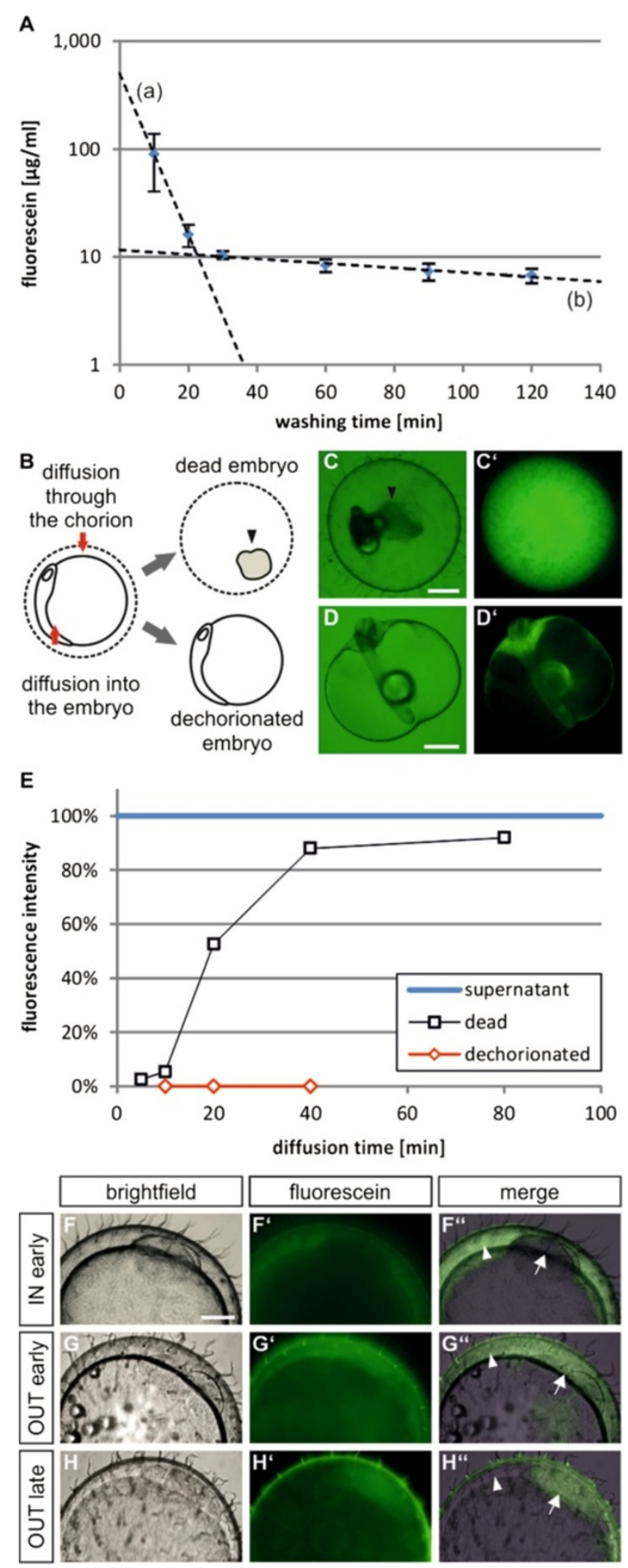

Figure $\mathbf{2}$ (See legend on next page.) 
(See figure on previous page.)

Figure 2 Diffusion barriers within the medaka egg. Stage 17 embryos were incubated with $10 \mathrm{mg} / \mathrm{ml}$ fluorescein for 40 minutes at $27^{\circ} \mathrm{C}$ and internal concentrations were determined at different time points of washing (A; mean values of 8-10 eggs are shown +/- SEM). (A) Dotted lines indicate the bi-phasic out-diffusion: (a) fast loss of the signal (first 2 measuring points), (b) slower second phase (best-fit of last 4 measuring points). (B, left side) shows a schematic view of an egg and the consecutive diffusion steps (red arrows). (C) Bright field picture of a dead embryo, representing diffusion through the chorion; the black arrow head indicates the shrunken embryo/yolk with the surrounding membranes. (D) Dechorionated embryo at stage 22 in dorsal view with anterior being at the top, $\left(\mathbf{C}^{\prime}, \mathbf{D}^{\prime}\right)$ corresponding fluorescent images. (E) Concentrations of fluorescein in dead or dechorionated embryos after different incubation times. The concentration of the supernatant (blue) was set to $100 \%$. Several measuring points (7,20 and $30 \mathrm{~min}$ ) were taken and the initial concentration at the onset of the washing calculated by extrapolation (compare A). (F-H) Eggs in brightfield, fluorescence and merged pictures. (F-F") Distribution of fluorescein after 10 minutes of in-diffusion (IN early) and 2 minutes of washing (1 mg/ml fluorescein). (G-G") Out-diffusion after 40 minutes of incubation (10 mg/ml fluorescein) and 5 minutes

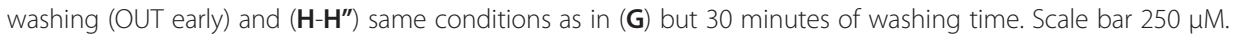

disruption of the fragile membranes). Therefore, not the chorion, but membrane systems within the medaka egg represent the main diffusion barrier for small molecules. These data would predict that diffusion of small molecules into intact embryos should rapidly proceed through the chorion into the perivitelline space, but would then be stopped at inner membranes. To test this hypothesis also with life embryos, we looked at eggs shortly exposed to fluorescein (10 minutes), which should be sufficient to reach high signal levels in the perivitelline space, but not in the embryo. Indeed, after a short washing step (2 minutes) the expected distribution was visible (Figure 2F-F"). Based on the previous experiments (Figure 1), the cells of the embryo should however show a signal when exposed for longer times (40 minutes). After a short washing step fluorescein should therefore be detectable in both the perivitelline space and the embryo (Figure 2G-G"; white arrowhead and arrow, respectively). During extended washing fluorescein should rapidly disappear from the perivitelline space (fast out-diffusion), but a signal would be retained in the embryo (slow out-diffusion). The expected results could be observed (Figure $2 \mathrm{H}-\mathrm{H}$ "). In order to verify this model also with another small molecule we tested methylene blue. As seen for fluorescein, methylene blue rapidly (after 10 minutes of incubation) accumulated in the perivitelline space (Additional file 2A). Longer washing times (20 minutes) quickly removed methylene blue again, but no signals were detectable in the cells of the embryo (Additional file 2B).

An internal diffusion barrier is also in perfect agreement with the bi-phasic out-diffusion profile (Figure 2A). The first phase represents loss of fluorescein from the perivitelline space (line a) and the second phase (line b) is caused by the considerably slower diffusion out of the embryo/yolk (resulting in a low steady state concentration in the perivitelline space). The half-time calculated for the initial out-diffusion (4 minutes) roughly fits to that of in-diffusion through the chorion (20 minutes; a factor of 5 differing the values can be explained by the larger interior volume of dead embryos compared to that of the perivitelline space). Therefore, the slow diffusion of small molecules into medaka embryos is caused by membrane systems close to the embryo and dechorionation does not improve the uptake.

\section{Detergents affect membrane behaviour}

In an attempt to overcome this diffusion barrier, we first focussed on the injection of the small molecules. Injection into single cells of the embryo is well established for early stages of development, leading to a rapid distribution in all cells of the embryo. During later development injection into large internal cavities, like the neural tube, would be an option. However this is again limited to certain stages. In order to accomplish uniform diffusion into the embryos, we tested injection into the yolk, a method that is often performed in zebrafish. Within 30 minutes the injected fluorescein evenly distributed throughout the yolk (Additional file 3A-C and F-G). However, the detection of signals in the embryo was only possible after 4 hours (Additional file 3D,K) and the obtained concentrations were low. Furthermore, yolk injection in medaka results in low reproducibility, due to rapid clogging of the injection needles by the sticky yolk.

Next we tested incubation of the embryos with detergents. Contrary to ionic detergents like SDS, which appeared highly toxic, Triton X-100 worked well. The embryos tolerated concentrations up to $0.01 \%$ (40 minutes incubation) without showing phenotypes. At $0.03 \%$ Triton $\mathrm{X}-100$, the survival was reduced to $69 \%$ and for $0.1 \%$ Triton to $25 \%$, but no obvious phenotypes were detectable in the surviving embryos. Co-incubation with $0.03 \%$ Triton $\mathrm{X}-100$ increased the fluorescein uptake of the eggs by a factor of 6 compared to control embryos treated with ERM (Figure 3A,D; 30 minutes of washing; note that different exposure times were used for the pictures). Interestingly, extended washing did not reduce the intensity of the fluorescent signal of detergent treated embryos (Figure 3D-F), indicating that the dye became trapped in the embryo/yolk. The signal intensity therefore increased to 20 fold after 60 minutes and to more than 80 fold after 24 hours (Figure 3B,E and C,F) compared to the control embryos at the same time points. Furthermore, Triton-treated eggs showed signals throughout the embryo 


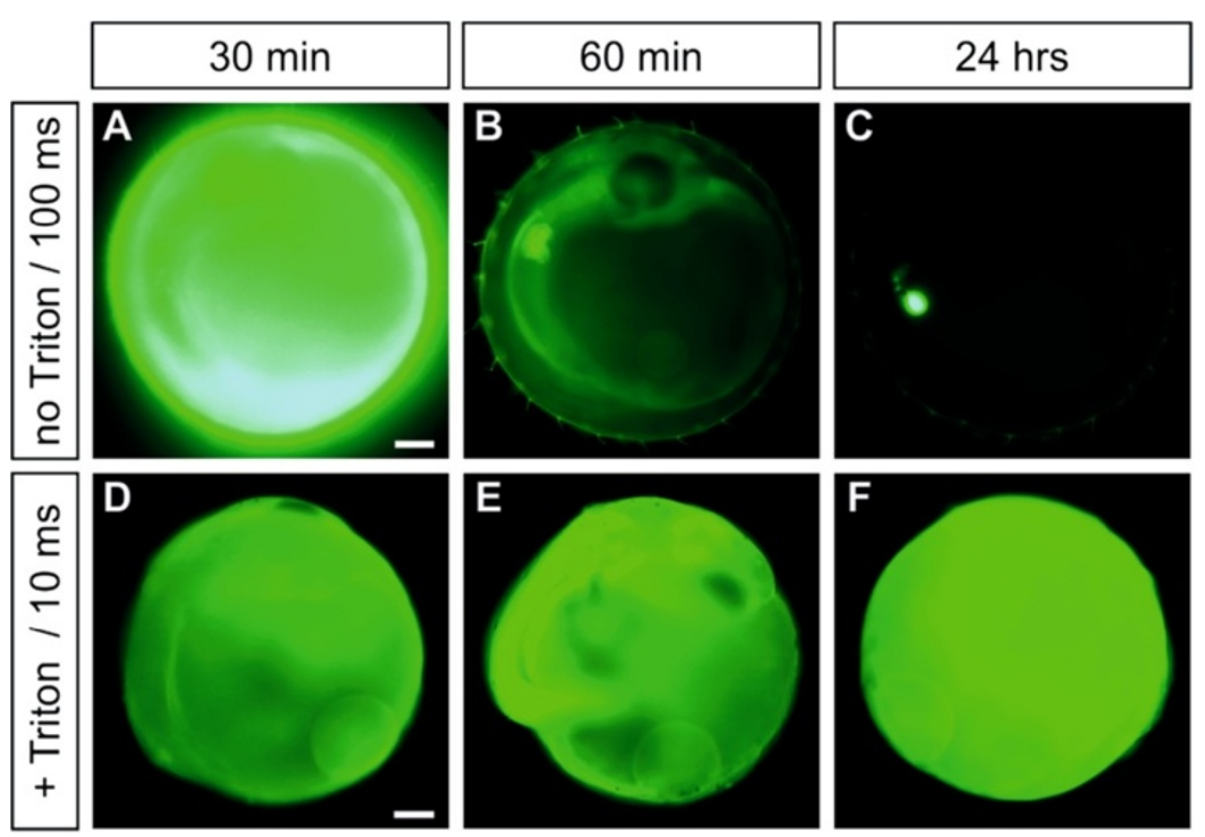

Figure 3 Incubation with detergents. 3 day old embryos incubated for 40 minutes with fluorescein (10 mg/ml) at different time points of washing without addition (A-C; no Triton), or together with $0.03 \%$ Triton X-100 (D-F; + Triton). Embryos are shown in lateral view, anterior to the top. Note that pictures (A-C) were taken with $100 \mathrm{~ms}$ exposure time, whereas (D-F) with $10 \mathrm{~ms}$. The accumulation of fluorescein in the liver/ gallbladder at later stages can be seen in (C). Scale bar $250 \mu \mathrm{M}$.

and the yolk (Figure 3D-F), whereas fluorescein was clearly enriched in the embryos of non-treated eggs (Figure 3B and Figure 1A-D). Therefore, addition of Triton X-100 improves the uptake of small molecules into the egg, but also affects their distribution between compartments, making the assessment of pharmacologic/toxicologic properties difficult.

\section{Electroporation improves small molecule uptake into embryos}

Another method for enhanced transport across membranes is electroporation. Hostetler and colleagues used direct current-shifted radio frequency pulses to generate transgenic medaka fish [20]. We used the same conditions as a starting point (modulation frequency, $35 \mathrm{kHz}$; voltage, $25 \mathrm{~V}$; pulse duration, $10 \mathrm{~ms}$; using 3 pulses with 1 second pulse interval) and measured the fluorescence intensity of fluorescein in electroporated stage $17 \mathrm{em}$ bryos. Due to the fact that fluorescein readily passes the chorion, we incubated the eggs prior to electroporation for 40 minutes with the staining solution. As a result high concentrations of the dye accumulated in the perivitelline space (compare Figure 2F"). To overcome the rate limiting membrane systems we applied electric pulses varying the voltage and pulse duration over a wide range. Finally, we obtained optimal fluorescence intensity and survival at $15 \mathrm{~V}$ and 5 seconds pulse duration (Figure 4A and Additional file 4). In contrast to
Hostetler and colleagues, these conditions represent a single pulse of extended length, but with comparable low voltage. Additional pulses did not improve fluorescein uptake into the embryos. Nevertheless, other combinations, such as high voltage with short pulses also resulted in significantly higher fluorescence intensity than diffusion alone. However, the combination of long pulses with high voltage was impossible to apply due to a reduced survival rate. The same conditions were also applicable for younger (Figure 4B') and older embryos (Figure 4C').

In order to verify that electroporation improves the rate limiting diffusion through inner membrane systems, we performed the same experiments with dead and dechorionated embryos (Figure 5). Electroporation did not change the signal intensity of fluorescein in dead embryos, indicating that it has no effect on the uptake through the chorion (Figure 5A,C,F), whereas dechorionated embryos showed a nearly 15-fold increase of dye intensity after electroporation (Figure 5A,D,G). Therefore, similar to whole embryos (Figure 5A,B,E), the dechorionated embryos still contain membranes acting as diffusion barriers, which can be made permeable by electroporation.

The electroporation experiments presented so far had been performed with modulation frequencies of $35 \mathrm{kHz}$. We next varied this frequency and found considerable differences in signal intensity. The optimum for fluorescein uptake appeared at $330 \mathrm{~Hz}$ (Figure 6A). In order to 

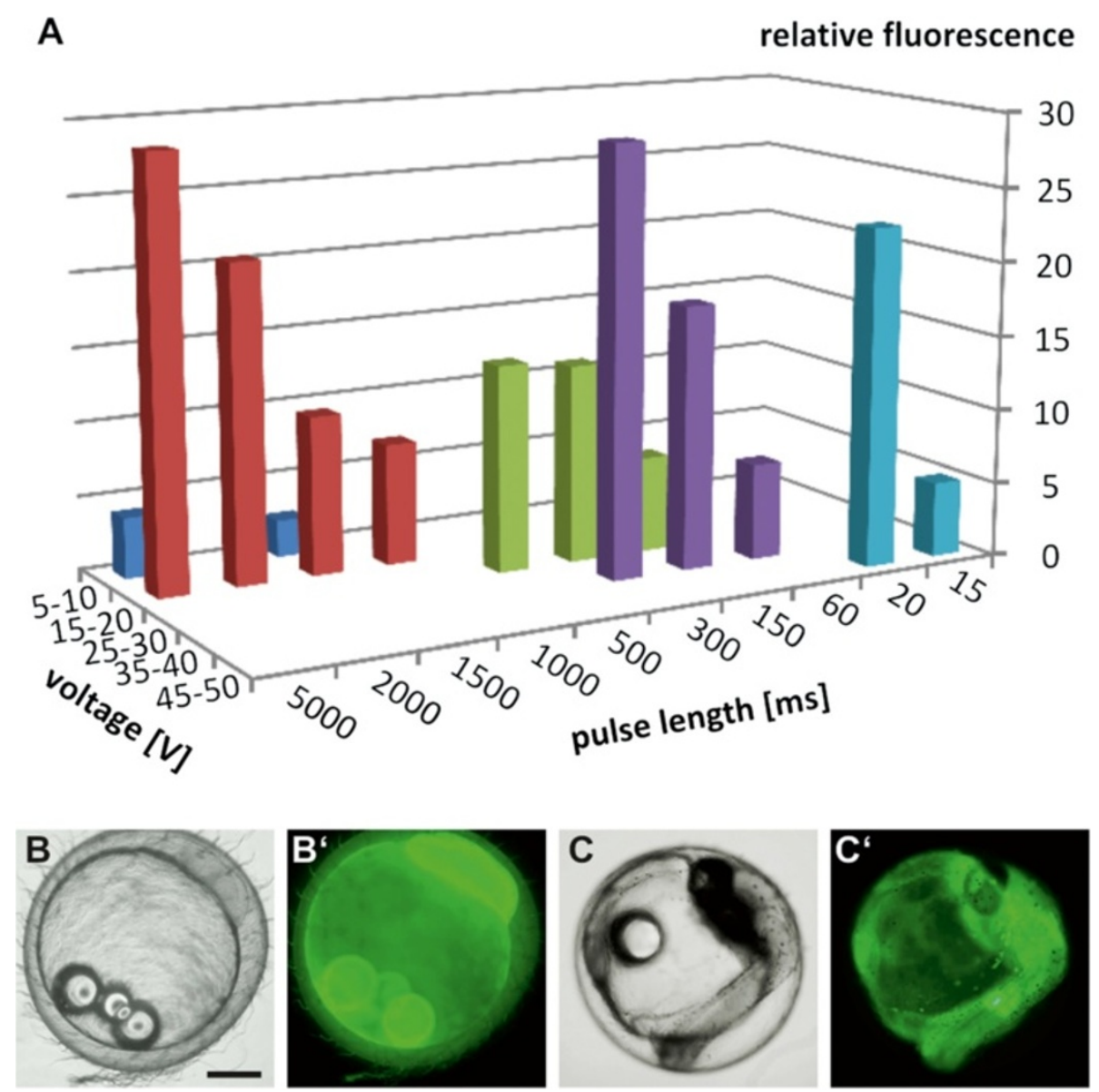

Figure 4 Optimization of electroporation conditions. (A) Embryos at stage 17 were incubated with $10 \mu \mathrm{g} / \mathrm{ml}$ fluorescein for 40 minutes at $27^{\circ} \mathrm{C}$. Subsequent electroporation was performed at $35 \mathrm{kHz}$ with varying voltages and pulse lengths. Relative fluorescence indicates fold quantified signal compared to that of non-electroporated, but otherwise equally treated reference embryos. Similar results were obtained for early (B, B', stage 4) and late (C, $\mathbf{C}^{\prime}$, stage 31) embryos. Embryos are shown in lateral (B, $\left.\mathbf{B}^{\prime}\right)$ and dorso-lateral $\left(\mathbf{C}, \mathbf{C}^{\prime}\right)$ view. Scale bar $250 \mu \mathrm{M}$.

extend the results to other small molecules, we selected two additional dyes (rhodamine $\mathrm{B}$ and acridine orange) which differ in their molecular properties from fluorescein. Thus rhodamine B becomes strongly enriched in the yolk (Figure 6B-D). Contrary to most other dyes we tested, washing only partly removed the dye from the egg, where it remained trapped in the yolk. Acridine orange showed a more uniform distribution being present both in the yolk and the embryo (Figure 6E-G). At later stages, strong fluorescence was observed in the gallbladder/ liver (Figure 6G). It therefore exhibits properties inbetween fluorescein and rhodamine B. As for fluorescein, we performed electroporation experiments with rhodamine $\mathrm{B}$ and acridine orange. Compared to diffusion alone electroporated embryos always showed an extended uptake of the molecules, demonstrating the potential of electroporation also for other small molecules. Taken together, electroporation enhances the uptake of different small molecules into medaka embryos.
Lithium induces deficiencies in anterior-posterior development

After the analysis of the diffusion properties of medaka eggs and the establishment of electroporation as a tool to improve the uptake, we tested the procedure for our initial experiments with lithium chloride. For this we used 0.4 M lithium chloride, a concentration comparable to the one used in zebrafish experiments [13]. Also the incubation time (10 minutes) was in agreement with the zebrafish experiments. Diffusion alone at stage 14 (40\% epiboly) resulted in normal development (Figure 7A). However, more than $60 \%$ of the embryos that were electroporated (15 V, $330 \mathrm{~Hz}, 100 \mathrm{~ms}, 1$ pulse) in the presence of lithium chloride showed clear deficiencies in anterior-posterior development (Figure 7A), with 24\% developing a weak phenotype and 39\% a strong phenotype (Figure 7A and Additional file 5). As a strong phenotype we classified all embryos that showed severe axis truncation anterior to the midbrain, resulting in missing eyes and forebrain (Figure 7D,G), whereas in weak 

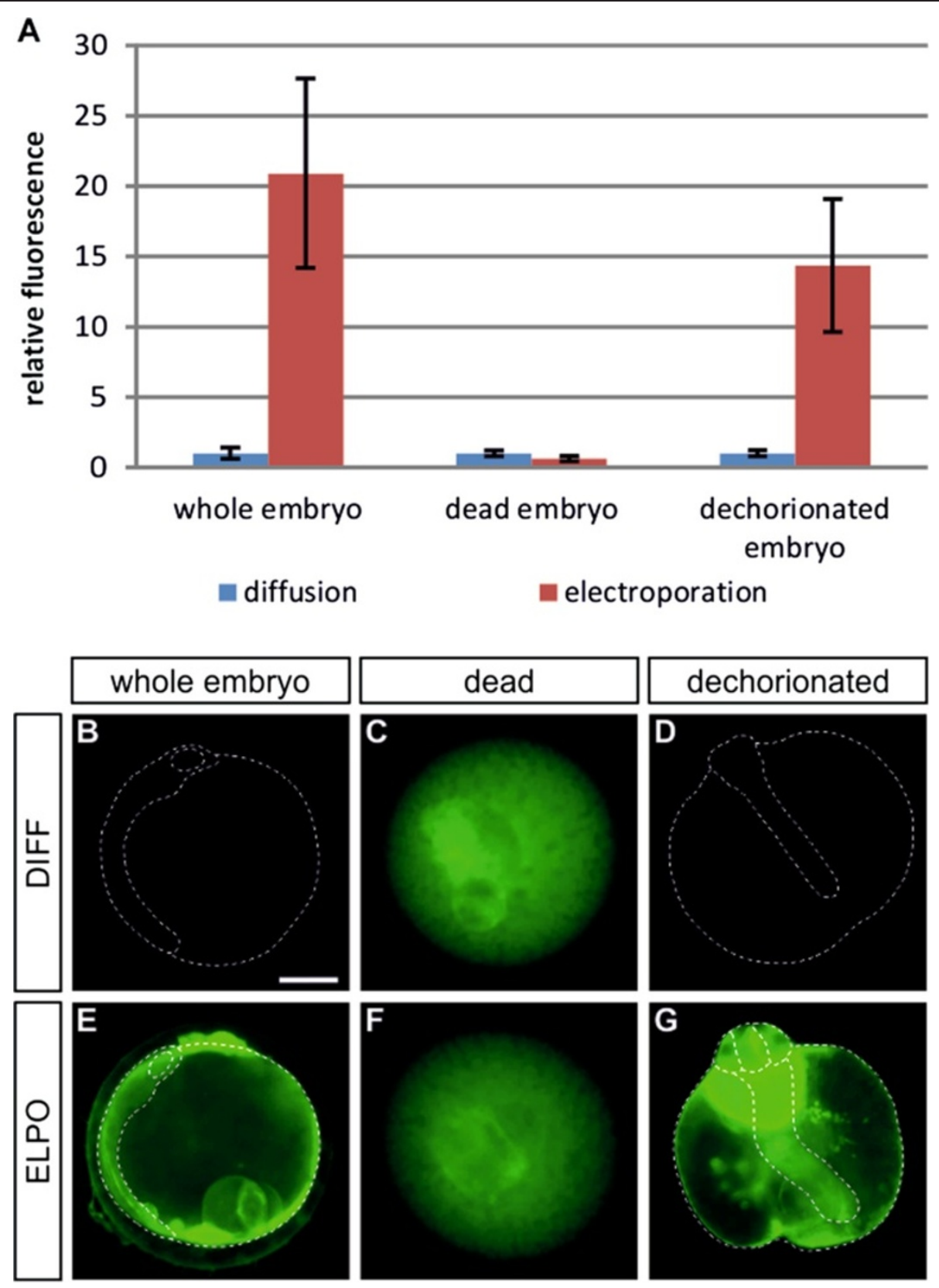

Figure 5 Electroporation affects the transfer of fluorescein through inner membranes, but not the chorion. Embryos were incubated with $10 \mathrm{mg} / \mathrm{ml}$ fluorescein for 40 minutes (A, blue bars; B-D); subsequent electroporation (A, red bars; E-G) was performed using $35 \mathrm{kHz}, 15 \mathrm{~V}$, $5000 \mathrm{~ms}$, 1 pulse. Pictures were taken after 30 minutes washing time. Embryos are shown in lateral $(\mathbf{B}, \mathbf{E})$ and dorsal $(\mathbf{D}, \mathbf{G})$ view with anterior to the top. Scale bar $250 \mu \mathrm{M}$. Abbreviations: ELPO, electroporation; DIFF, diffusion.

phenotypes only the eyes were affected (Figure 7C,F). Furthermore, we observed the formation of enlarged and ectopic otic vesicles (Additional file 6), which again is in good agreement with the phenotypes seen for wnt1 overexpression [17]. Control embryos that were electroporated and not treated with lithium chloride developed normally, but showed an elevated mortality rate (Figure 7A and Additional file 5). Nevertheless, electroporation could be established as a tool to enhance the uptake of small molecules into medaka embryos in order to study effects on development.

\section{Discussion}

Several studies have been performed to qualify the effect of small molecules on fish embryos [21-23]. In addition, toxicological applications like the FET test are discussed as an alternative to experiments with mammals and birds [8]. The uptake of substances by the embryos has 

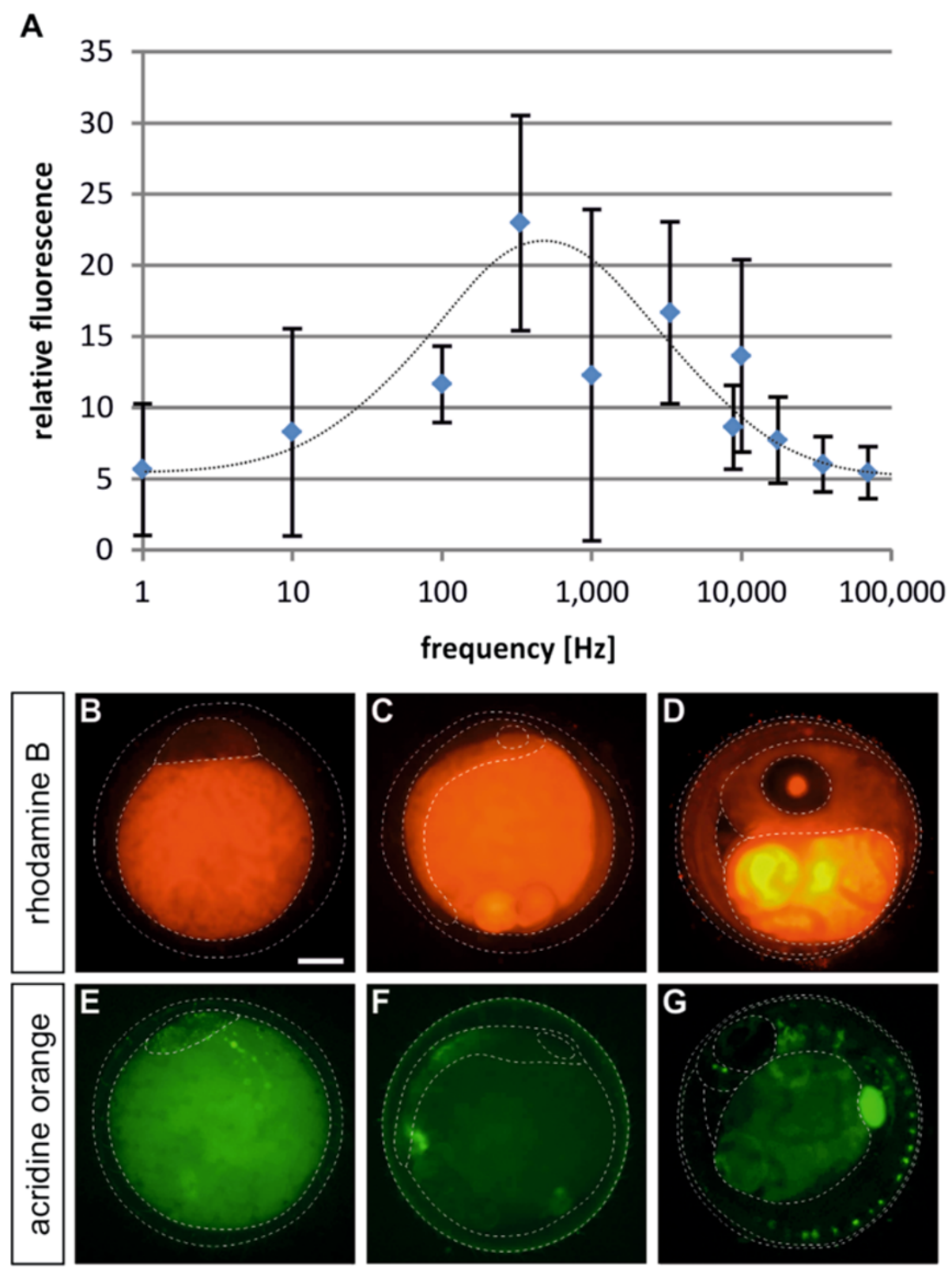

Figure 6 Electroporation of rhodamine B and acridine orange. (A) Optimisation of the modulation frequency of electroporation. The electroporation was performed at $15 \mathrm{~V} ; 5000 \mathrm{~ms} ; 1$ pulse using variable frequencies. Relative fluorescence indicates fold fluorescein signal measured compared to non-electroporated, but otherwise equally treated reference embryos. For extension to other small molecules, the embryos were incubated with $10 \mathrm{ng} / \mathrm{ml}$ rhodamine $\mathbf{B}(\mathbf{B}-\mathbf{D})$ and $1 \mu \mathrm{g} / \mathrm{ml}$ acridine orange $(\mathbf{E}-\mathbf{G})$ at stage $2 \mathrm{a}(\mathbf{B}, \mathbf{E})$, stage $17(\mathbf{C}, \mathbf{F})$ and stage $36(\mathbf{D}, \mathbf{G})$ for 40 minutes at $27^{\circ} \mathrm{C}$. Electroporation was performed using $330 \mathrm{~Hz} ; 15 \mathrm{~V} ; 5000 \mathrm{~ms} ; 1$ pulse. The dotted lines demarcate the outlines of: (B,E) yolk and blastoderm, and $(\mathbf{C}, \mathbf{D}, \mathbf{F}, \mathbf{G})$ yolk and embryo. Embryos are shown in lateral view. Scale bar $250 \mu \mathrm{M}$.

been recognized as a critical factor in these experiments [24,25]. However, little is known about the diffusion properties of the chorion and other membrane systems covering fish embryos. We used fluorescing substances as a model to study the diffusion of small molecules into medaka embryos. Combined with fluorescence microscopy quantification this offers a number of advantages over conventional techniques like radioactive labelling.
Firstly, individual embryos can be followed over the time and quantified repeatedly. Secondly, the internal distribution of the molecules in the embryo due to the physicochemical nature of the molecules can be examined and compared at different stages of development. Alterations in the compartment specific distribution, as for example after incubation with detergents (Figure 3), are highly important for the assessment of pharmacokinetic 

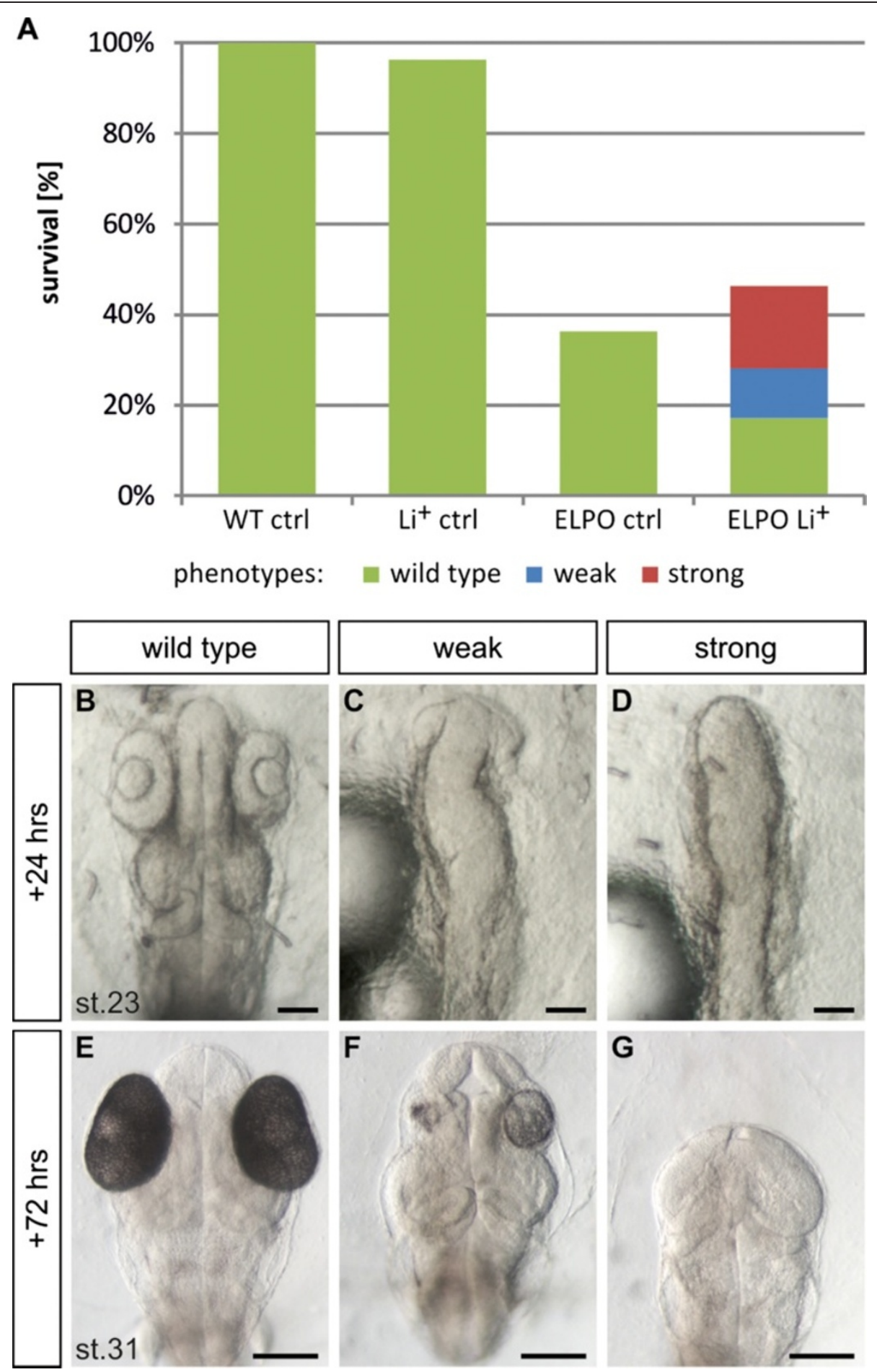

Figure 7 Lithium induction causes deficiencies in anterior-posterior development. Embryos at $40 \%$ epiboly were exposed to $0.4 \mathrm{M}$ LiCl for 10 minutes at $27^{\circ} \mathrm{C}$. Subsequent electroporation was performed at $330 \mathrm{~Hz} ; 15 \mathrm{~V} ; 100 \mathrm{~ms} ; 1$ pulse. 24 hours after the induction (+24 hrs) the phenotypes were clearly detectable and finally categorized after 72 hours ( $+72 \mathrm{hrs}$ ) as: wild type (green; B, E), weak (blue; C, F) and strong phenotypes (red; D, G). (A) The control $\left(\mathrm{Li}^{+} \mathrm{Ctrl}\right.$ ) shows equally treated but non-electroporated embryos, electroporation control embryos (ELPO ctrl) were electroporated, but not exposed to LiCl. Electroporated embryos with LiCl (ELPO Li+) showed phenotypes indicated by colours. Embryos are shown in dorsal view with anterior to the top. Scale bar $100 \mu \mathrm{M}$. 
properties of drugs. Thirdly, metabolic processing can be followed (e.g. as for the accumulation of fluorescein and acridine orange in the liver/gallbladder). And finally, the distribution in different compartments due to variable diffusion rates can be detected, as the accumulation in the perivitelline space (Figure $\left.2 \mathrm{~F}-\mathrm{H}^{\prime \prime}\right)$.

We used fluorescein, rhodamine $\mathrm{B}$, acridine orange, and lithium chloride for our experiments. They represent small molecules which cover a large spectrum of different properties from charged (lithium chloride) to non-charged (acridine orange). The substances exhibited varying distributions in medaka eggs, indicating differences in their hydrophobic properties (rhodamine B being most strongly enriched in the yolk). Nevertheless, all substances showed low diffusion rates into medaka embryos. In contrast to zebrafish, medaka eggs contain a hard chorion. Based on suggestions from the literature [3], we therefore suspected the chorion to represent a diffusion barrier, shielding the embryos from their chemical environment. Following sperm entry, a calcium wave moves along the surface and the chorion matures [26], thereby reaching maximum hardness approximately 6 hours after fertilization [12]. We therefore expected a dramatic reduction of the diffusion rate after chorion hardening. However, no differences appeared in the experiments (Figure 1E). As the chorion permeability is connected to calcium ions $[27,28]$ we incubated the embryos right after fertilization in a calcium-free medium [29], with different $\mathrm{pH}$ values and even tried incubation in distilled water. However, the uptake of fluorescein into the embryos did not improve (data not shown). Therefore, conditions affecting chorion hardening did not alter the diffusion rates into the egg, neither did dechorionation of the embryos (Figure 2D', E). Instead we observed a rapid diffusion through the chorion into dead embryos (Figure 2C'.E). The fact that inner membrane systems and not the chorion represent the main diffusion barrier in medaka eggs was further supported by the fast accumulation of signals in the perivitelline space (early in-diffusion; Figure 2F-F") and the rapid loss of this signal during out-diffusion (Figure 2G-H"). On the contrary, accumulation in the embryo needed extensive in-diffusion times, but once achieved stayed considerably longer than that of the perivitelline space (Figure 2F-H"). The result is a bi-phasic progression of diffusion (Figure 2A), caused by a strong diffusion barrier positioned closer to the embryo than the chorion. During blastula stage, the egg can be subdivided into three distinct cell lineages. One lineage consists of the pluripotent deep layer blastomeres (DEL), which produce the future embryo proper. The second domain consists of a syncytium of multiple nuclei, called the yolk syncytial layer (YSL). The third domain is the envelope layer (EVL), a thin cell layer which covers the DEL and will eventually form the periderm [30]. Both the second and the third domain are extra-embryonic. According to our results, the EVL/ periderm would be a candidate to block the diffusion into the egg.

These results have important implications for pharmacologic and toxicologic experiments with medaka embryos. Due to the bi-phasic diffusion process, the substance is initially enriched in the perivitelline space (diffusion halftime in the range of a few minutes). Subsequently, the slow diffusion into the embryo/yolk starts (diffusion halftime in the range of several hours). Therefore extended exposure to the test substances is necessary to evaluate pharmacologic/toxicologic consequences on embryonic development. Due to the slow accumulation in the embryo, effects on the first hours of development (e.g. early teratogenic properties) will not be detectable without enhanced transfer.

Assuming that the main diffusion barrier is a membrane system, it should be possible to increase its permeability by addition of detergents. Indeed, addition of Triton X-100 improved the uptake of fluorescein dramatically, but severely affected the membrane systems of the embryo (see Figure 3). Also injection into the yolk turned out to be inefficient. A possibility to make membranes permeable is electroporation. We applied current shifted radio frequency pulses [20] and could optimize the conditions to substantially increase the dye uptake compared to diffusion alone. Also other electroporation techniques like nanosecond pulsed electric fields have been tested for this purpose [31]. Such techniques would not only be of interest for small molecules, but also for the transfer of DNA or RNA. Indeed, Hostetler and colleagues initially proposed this technique to obtain transgenic medaka fish. In our hand the method worked effectively for small molecules, however, we failed with DNA. Closer inspection revealed that not even fluorescence labelled oligonucleotides (MW 6000) could pass the chorion, suggesting a size exclusion for the pores of the chorion in that range (data not shown). Electroporation did not improve this transfer. We also used DNA expression constructs containing gfp and luciferase for more sensitive assays, but failed to detect any marker gene expression in intact electroporated embryos. Only after dechorionation we could successfully transfer DNA into the embryos by electroporation (detection of gfp expression; data not shown). However, the survival of dechorionated embryos is extremely low during electroporation, making the method inapplicable for routine experiments. Injection of DNA into the perivitelline space and subsequent electroporation would be an option. However, direct injection of DNA into the zygote represents a simple alternative.

Having established electroporation for the uptake of small molecules into the living medaka embryo, we then 
returned to our initial question, the application of lithium to medaka development. The effects of lithium on developmental processes have been shown in several organisms. Already more than 60 years ago it was known that amphibian embryos developed severe anterior truncations when exposed to lithium during gastrulation (reviewed in [18]). This dorsalization effect was explained by the finding that lithium inhibits GSK-3 $\beta$, a key component of the Wnt signalling pathway [32,33]. Indeed, diffusion and subsequent electroporation led to axis truncations in medaka embryos, similar to those observed upon ectopic expression of wnt1 [17]. The embryos either failed to develop anteriorly to the midbrain (Figure 7D,G), lacking eyes and the forebrain (strong phenotype), or they developed eyes with reduced size (Figure 7C,F; weak phenotype). Electroporation therefore effectively improves the transfer of small molecules into medaka embryos. Hence, this method can be used to enhance the uptake of chemicals. Examples of such applications are specific inducers or repressors of signalling pathways to study embryonic development. For these experiments timing represents an important parameter. Slow diffusion and consequently slow accumulation prevents an exact timing of the effective concentration in the embryo. Electroporation strongly increases the internal concentration within a single step as exemplified for lithium chloride (the timing of Wnt signalling pathway activation critically affects the observed phenotypes; [17]).

\section{Conclusion}

During our experiments we found that membrane layers surrounding the medaka embryo represent a diffusion barrier for small molecules, whereas the hard outer chorion is readily passed. The slow diffusion has to be considered when toxicologic or pharmacologic experiments are performed with medaka embryos. A possible way to overcome this problem is electroporation, which substantially improves the uptake of small molecules. We thus were able to induce medaka embryos with the GSK-3 inhibitor lithium and could show that the resulting activation of the Wnt signalling pathway causes deficiencies in anterior-posterior development.

\section{Additional files}

Additional file 1: Fluorescein enrichment in the gallbladder/liver.

Embryos at the 1-cell stage were incubated with $10 \mathrm{mg} / \mathrm{ml}$ fluorescein for 40 minutes at $27^{\circ} \mathrm{C}$. Standard washing steps were performed and pictures were taken after 3 days (stage 31). Embryos are shown in lateral view with anterior to the left. (A) Fluorescence and (B) bright field pictures of the eggs, arrow heads indicate staining in the gallbladder. Scale bar $250 \mu \mathrm{M}$.

Additional file 2: Methylene blue diffusion into embryos. Embryos at the 1-cell stage were incubated with $0.001 \%$ methylene blue in $10 \times$ ERM for 10 minutes at $27^{\circ} \mathrm{C}$. Standard washing steps were performed and brightfield pictures were taken after 10 (A) and 20 minutes (B) of washing. Embryos are shown in lateral view; the blastomeres are on the top. Scale bar $250 \mu \mathrm{M}$. Abbreviations: DIFF, diffusion.

Additional file 3: Yolk injection. Embryos at the 1-cell stage (upper row) and stage 30 (lower row) were injected with $10 \mathrm{mg} / \mathrm{ml}$ fluorescein. Embryos are shown in lateral view, blastomeres (A-D) or anterior (E-J) to the top right. The yolk and embryo are demarcated by the dotted lines, the outlines of the chorion by continuous white lines. Incubation time after yolk-injection is indicated by the time designation at the bottom left. In both early (D) and late (I) yolk-injected embryos weak fluorescence ( $D$; arrow head) was only detected after 4 hours within the embryo. Older embryos showed clear enrichment of fluorescein within the gallbladder/liver (I,j; arrow). Scale bar $250 \mu \mathrm{M}$. Abbreviations: min, minutes; hrs, hours.

Additional file 4: Results of electroporation optimization experiments. Embryos at stage 17 were incubated with $10 \mu \mathrm{g} / \mathrm{ml}$ fluorescein for 40 minutes at $27^{\circ} \mathrm{C}$. Subsequent electroporation was performed at $15 \mathrm{kHz}$ with varying voltages and pulse lengths. Fluorescence intensity is shown in the top row and was normalized to the values measured for the diffusion control. Survival: percentage of surviving embryos after 20 minutes of washing. In order to obtain more significant results, experiments with similar conditions (voltage) were combined.

Additional file 5: Electroporation of lithium incubated embryos. Embryos at 40\% epiboly were incubated with $0.4 \mu \mathrm{M}$ lithium chloride for 10 minutes at $27^{\circ} \mathrm{C}$. Thereafter, embryos were either directly transferred into ERM (Diffusion), or electroporation was performed at $330 \mathrm{~Hz}, 15 \mathrm{~V}$, $100 \mathrm{~ms}$ using a single pulse (Electroporation). Phenotypes were categorized into strong (eyes were missing) and weak (only small eyes

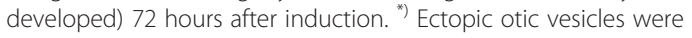
observed only in embryos developing a strong phenotype and were not considered in the calculation of the percentage of phenotypes in surviving embryos.

Additional file 6: Ectopic otic vesicles in lithium induced embryos. Embryos at $40 \%$ epiboly were exposed to $0.4 \mathrm{M} \mathrm{LiCl}$ for 10 minutes at $27^{\circ} \mathrm{C}$ followed by electroporation at $330 \mathrm{~Hz} ; 15 \mathrm{~V} ; 100 \mathrm{~ms} ; 1$ pulse (longer pulse lengths resulted in reduced survival of the embryos). Embryo is shown in dorsal view with anterior to the top 72 hours after the induction, black arrowheads indicate ectopic otic vesicles. Scale bar $100 \mu \mathrm{M}$.

\section{Competing interests}

The authors declare that they have no competing interests.

\section{Authors' contributions}

GJ carried out the majority of diffusion experiments, the electroporation experiments with lithium and drafted the manuscript. MH performed all other electroporation experiments. AF participated in the diffusion experiments. $\mathrm{CH}$ and JW build the electroporation apparatus. TC was responsible for coordination of the experiments and writing of the manuscript. All authors read and approved the final manuscript.

\section{Acknowledgement}

The work was supported by the Austrian Science Fund (FWF, grant P19571B11) and the City of Vienna (MA23 - project 10-20).

\section{Author details}

'Department for Applied Life Sciences, University of Applied Sciences, FH Campus Wien, Helmut-Qualtinger-Gasse 2, A-1030, Vienna, Austria.

${ }^{2}$ Department for Engineering, University of Applied Sciences, FH Campus Wien, Favoritenstrasse 226, A-1100, Vienna, Austria.

Received: 14 February 2013 Accepted: 17 June 2013

Published: 1 July 2013

\section{References}

1. Wittbrodt J, Shima A, Schartl M: Medaka-a model organism from the far East. Nat Rev Genet 2002, 3(1):53-64. 
2. Pastva SD, Villalobos SA, Kannan K, Giesy JP: Morphological effects of Bisphenol-A on the early life stages of medaka (Oryzias latipes). Chemosphere 2001, 45(4-5):535-541.

3. Villalobos SA, Hamm JT, Teh SJ, Hinton DE: Thiobencarb-induced embryotoxicity in medaka (Oryzias latipes): stage-specific toxicity and the protective role of chorion. Aquat Toxicol 2000, 48(2-3):309-326.

4. Kordes C, Rieber EP, Gutzeit HO: An in vitro vitellogenin bioassay for oestrogenic substances in the medaka (Oryzias latipes). Aquat Toxicol 2002, 58(3-4):151-164

5. Kang IJ, Yokota H, Oshima Y, Tsuruda Y, Oe T, Imada N, Tadokoro H, Honjo T: Effects of bisphenol a on the reproduction of Japanese medaka (Oryzias latipes). Environ Toxicol Chem 2002, 21(11):2394-2400.

6. Scialli AR: The challenge of reproductive and developmental toxicology under REACH. Regul Toxicol Pharmacol 2008, 51(2):244-250.

7. Embry MR, Belanger SE, Braunbeck TA, Galay-Burgos M, Halder M, Hinton DE, Leonard MA, Lillicrap A, Norberg-King T, Whale G: The fish embryo toxicity test as an animal alternative method in hazard and risk assessment and scientific research. Aquat Toxicol 2010, 97(2):79-87.

8. Strahle U, Scholz S, Geisler R, Greiner P, Hollert H, Rastegar S, Schumacher A, Selderslaghs I, Weiss C, Witters $\mathrm{H}$, et al: Zebrafish embryos as an alternative to animal experiments-a commentary on the definition of the onset of protected life stages in animal welfare regulations. Reprod Toxicol 2012, 33(2):128-132

9. Lammer E, Carr GJ, Wendler K, Rawlings JM, Belanger SE, Braunbeck T: Is the fish embryo toxicity test (FET) with the zebrafish (Danio rerio) a potentia alternative for the fish acute toxicity test? Comp Biochem Physiol C Toxicol Pharmacol 2009, 149(2):196-209.

10. Braunbeck T, Boettcher $M$, Hollert H, Kosmehl T, Lammer E, Leist E, Rudolf M, Seitz N: Towards an alternative for the acute fish LC(50) test in chemical assessment: the fish embryo toxicity test goes multi-species - an update. ALTEX 2005, 22(2):87-102

11. Yang L, Ho NY, Alshut R, Legradi J, Weiss C, Reischl M, Mikut R, Liebel U, Muller F, Strahle U: Zebrafish embryos as models for embryotoxic and teratological effects of chemicals. Reprod Toxicol 2009, 28(2):245-253.

12. Yamagami K, Hamazaki TS, Yasumasu S, Masuda K, luchi I: Molecular and cellular basis of formation, hardening, and breakdown of the egg envelope in fish. Int Rev Cytol 1992, 136:51-92.

13. Stachel SE, Grunwald DJ, Myers PZ: Lithium perturbation and goosecoid expression identify a dorsal specification pathway in the pregastrula zebrafish. Development 1993, 117(4):1261-1274.

14. Iwamatsu T: Stages of normal development in the medaka Oryzias latipes. Mech Dev 2004, 121(7-8):605-618.

15. Schneider CA, Rasband WS, Eliceiri KW: NIH Image to ImageJ: 25 years of image analysis. Nat Methods 2012, 9(7):671-675.

16. Ortner V, Kaspar C, Halter C, Tollner L, Mykhaylyk O, Walzer J, Gunzburg WH, Dangerfield JA, Hohenadl C, Czerny T: Magnetic field-controlled gene expression in encapsulated cells. J Control Release 2012, 158(3):424-432.

17. Bajoghli B, Aghaallaei N, Jung G, Czerny T: Induction of otic structures by canonical Wnt signalling in medaka. Dev Genes Evol 2009, 219(8):391-398.

18. Kao KR, Elinson RP: The legacy of lithium effects on development. Bio/ Cell 1998, 90(8):585-589.

19. Schmidt R, Buscher HP: Hepatic uptake of fluorescein, investigated by video fluorescence microscopy and digital image analysis. J Hepatol 1991, 13(2):208-212

20. Hostetler HA, Peck SL, Muir WM: High efficiency production of germ-line transgenic Japanese medaka (Oryzias latipes) by electroporation with direct current-shifted radio frequency pulses. Transgenic Res 2003, 12(4):413-424

21. Modra H, Vrskova D, Macova S, Kohoutkova J, Hajslova J, Haluzova I, Svobodova Z: Comparison of diazinon toxicity to embryos of Xenopus laevis and Danio rerio; degradation of diazinon in water. Bull Environ Contam Toxicol 2011, 86(6):601-604

22. Osterauer R, Kohler HR: Temperature-dependent effects of the pesticides thiacloprid and diazinon on the embryonic development of zebrafish (Danio rerio). Aquat Toxicol 2008, 86(4):485-494.

23. Oxendine SL, Cowden J, Hinton DE, Padilla S: Adapting the medaka embryo assay to a high-throughput approach for developmental toxicity testing. Neurotoxicology 2006, 27(5):840-845.

24. Hamm JT, Wilson BW, Hinton DE: Increasing uptake and bioactivation with development positively modulate diazinon toxicity in early life stage medaka (Oryzias latipes). Toxicol Sci 2001, 61(2):304-313.
25. Harvey B, Kelley RN, Ashwood-Smith MJ: Permeability of intact and dechorionated zebra fish embryos to glycerol and dimethyl sulfoxide. Cryobiology 1983, 20(4):432-439.

26. Gilkey JC, Jaffe LF, Ridgway EB, Reynolds GT: A free calcium wave traverses the activating egg of the medaka. Oryzias latipes. J Cell Biol 1978 76(2):448-466

27. Masuda K, Luchi I, Yamagami K: Analysis of Hardening of the Egg Envelope (Chorion) of the Fish, Oryzias latipes. Dev Growth Differ 1991, 33(1):75-83.

28. Masuda K, Murata K, luchi I, Yamagami K: Some Properties of the Hardening Process in Chorions Isolated from Unfertilized Eggs of Medaka, Oryzias latipes. Dev Growth Differ 1992, 34(5):545-551.

29. Iwamatsu T, Shibata Y, Kanie T: Changes in chorion proteins induced by the exudate released from the egg cortex at the time of fertilization in the teleost, Oryzias latipes. Dev Growth Differ 1995, 37(6):747-759.

30. Kimmel CB, Warga RM, Schilling TF: Origin and organization of the zebrafish fate map. Development 1990, 108(4):581-594.

31. Tominaga N, Kono S, Yamaguchi A, Akiyama H, Arizono K: A novel material incorporation technique for medaka (Oryzias latipes) eggs using nanosecond pulsed electric fields. Biosci Biotechnol Biochem 2009, 74(6):1279-1282

32. Klein PS, Melton DA: A molecular mechanism for the effect of lithium on development. Proc Natl Acad Sci U S A 1996, 93(16):8455-8459.

33. Stambolic $V$, Ruel L, Woodgett JR: Lithium inhibits glycogen synthase kinase-3 activity and mimics wingless signalling in intact cells. Curr Biol 1996, 6(12):1664-1668

doi:10.1186/1472-6750-13-53

Cite this article as: Jung et al:: Diffusion of small molecules into medaka embryos improved by electroporation. BMC Biotechnology 2013 13:53.

\section{Submit your next manuscript to BioMed Central and take full advantage of:}

- Convenient online submission

- Thorough peer review

- No space constraints or color figure charges

- Immediate publication on acceptance

- Inclusion in PubMed, CAS, Scopus and Google Scholar

- Research which is freely available for redistribution
C Biomed Central 